\title{
Traduire
}

Une autre perspective sur r tr traduction

Revue française de la traduction

$243 \mid 2020$

Les arts du spectacle

\section{Le sous-titrage et le doublage au cinéma}

Entretien avec Maï Boiron

Maï Boiron et Émilie Syssau

\section{(2) OpenEdition}

Journals

Édition électronique

URL : http://journals.openedition.org/traduire/2101

DOI : 10.4000/traduire.2101

ISSN : 2272-9992

Éditeur

Société française des traducteurs

Édition imprimée

Date de publication : 15 décembre 2020

Pagination : 7-19

ISSN : 0395-773X

Référence électronique

Maï Boiron et Émilie Syssau, «Le sous-titrage et le doublage au cinéma », Traduire [En ligne], 243 |

2020, mis en ligne le 15 décembre 2020, consulté le 31 décembre 2020. URL : http://

journals.openedition.org/traduire/2101; DOI : https://doi.org/10.4000/traduire.2101 


\section{Le sous-titrage et le doublage au cinéma}

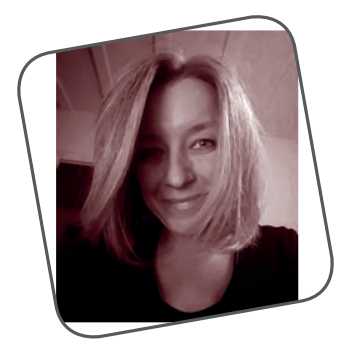

Entretien avec Maï Boiron

Quand on va au cinéma pour voir un film en langue étrangère, on peut choisir de le regarder dans sa version originale sous-titrée, ou doublé en français. L'une et l'autre requièrent en amont l'intervention d'un.e traducteur.trice', qui doit pour chacune mobiliser des compétences différentes et se conformer à diverses exigences. Écrire des sous-titres consiste à transposer un discours oral à l'écrit, à faire coexister à l'écran voix originales (entendues) et traduction textuelle (lve) de façon aussi discrète que possible. Le doublage a quant à lui vocation à proposer une adaptation des dialogues qui est substituée aux paroles prononcées par les acteurs. Mais comment procède-t-on dans chacun de ces cas? Maï Boiron, qui travaille presque exclusivement pour les majors américaines du cinéma, nous l'explique. 


\section{Les étapes du sous-titrage}

Je suis en général contactée par un.e directeur.trice ${ }^{2}$ technique de distribution, chargé.e de l'aspect technique des sorties de films en France, qui m'indique à quel laboratoire vont être confiées la pellicule et la spotting list, c'està-dire le dialogue découpé par les Américains, précisant les time codes (minutage) de chaque sous-titre. Le laboratoire choisi procède au repérage et prépare les cases où sera saisie la traduction, puis envoie un fichier informatique à introduire dans un logiciel de sous-titrage et un fichier texte: la spotting list précédemment mentionnée, ou la dialogue list - retranscription de l'intégralité des dialogues, assortie d'annotations expliquant certains aspects culturels et fournissant des éléments de jargon ou d'argot. Ces informations sont en général préparées par l'attaché.e de production après le tournage. C'est très confortable d'en disposer quand c'est bien fait.

Avant de commencer la traduction, il convient de regarder le film en entier. Il est fréquent de travailler sur une image préliminaire où les effets spéciaux ne sont pas terminés (on voit encore les fonds verts). Parfois, je reçois même le film au stade de story-board ${ }^{3}$. II arrive aussi que le montage change encore (coupures ou interversion de scènes, modification ou déplacement d'une voix off) ou que le mixage son n'ait pas été réalisé. On m’envoie les versions préliminaires successives P1, P2, P3, etc. - j'ai déjà eu jusqu'à sept versions différentes. Rien n'empêche de commencer la traduction sur un film inachevé, mais cela peut constituer une perte de temps. II vaut mieux attendre une version relativement définitive. Les vidéos dont je dispose sont souvent en noir et blanc, lardées d'inscriptions (noms du laboratoire et du chargé de production), en transparence, certes, mais l'image en est quand même brovillée.

L'élaboration d'un sous-titrage demande dix à quinze jours - c'est la durée idéale pour effectuer un premier jet, laisser

2. Par souci de concision, nous adopterons par la suite le générique «directeur».

3. Fichier numérique utilisé en préproduction afin de planifier les besoins de l'ensemble des plans qui constitueront le film, aussi bien au niveau technique (cadrages, mouvements de caméra, effets spéciaux) qu'au niveau artistique (décors construits, décors virtuels). 
reposer et relire. Je saisis la traduction dans le logiciel de sous-titrage aux endroits prévus par le laboratoire qui a découpé le dialogue, en respectant un nombre maximum de caractères pour chaque sous-titre, en fonction de sa durée: on ne peut évidemment pas lire la même quantité d'informations en une seconde qu'en quatre ou cinq (durée maximum d'un sous-titre). Une des contraintes du sous-titrage est en effet de respecter la lisibilité, à raison de quinze caractères par seconde en moyenne. Il faut alors trouver pour chaque phrase une version très synthétique. L'exercice consiste à condenser, à choisir, à "écrémer», dit-on souvent, pour ne garder que l'essentiel de l'information à transmettre. On reproche souvent au sous-titreur de ne pas tout traduire, mais c'est dans l'intérêt du spectateur. Certes, il arrive de perdre des nuances par rapport à l'original, mais si l'on traduisait tout, ce serait illisible. Par ailleurs, certains éléments passent par le ton, le contexte, l'image: le spectateur voit les situations à l'écran et peut, même inconsciemment, compléter le texte du sous-titre. En écoutant l'original, il peut aussi reconnaître des mots, a fortiori pour un film en anglais. II est davantage dépendant du sous-titre pour d'autres langues moins pratiquées - mais il a l'avantage d'entendre l'émotion originale.

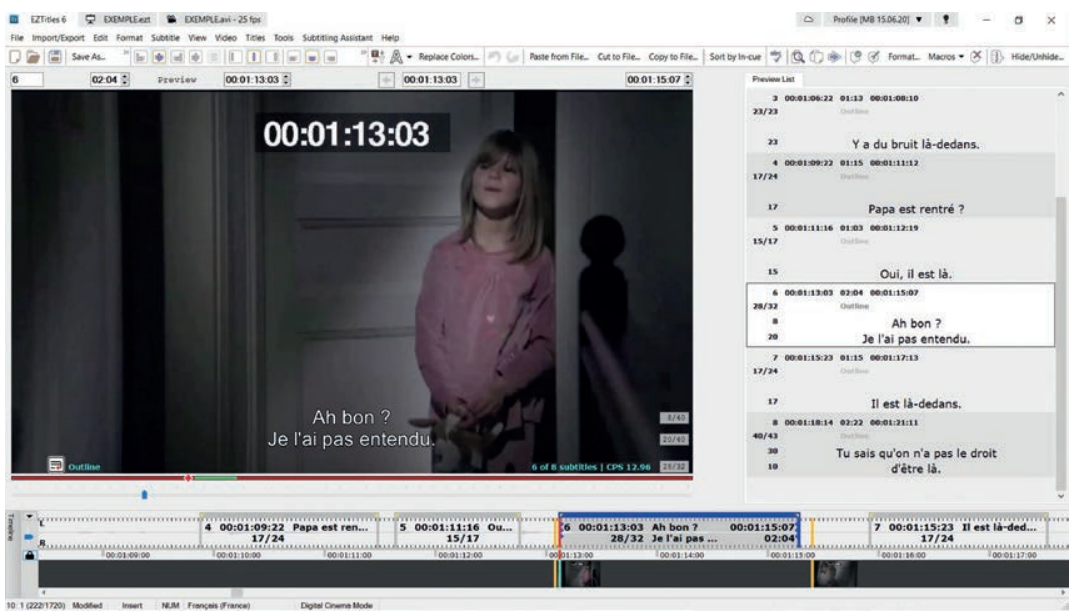

Interface de travail pour le sous-titrage. 
Le temps d'élaboration du sous-titrage varie d'un long métrage à l'autre: certains sont plus simples que d'autres, plus bavards, plus longs. Le sujet a par ailleurs son importance: dès lors qu'il est très spécialisé, il nécessite d'importantes recherches documentaires. Je fais parfois appel à un expert, comme pour First man ${ }^{4}$, sur la mission Apollo 11, qui intègre des dialogues historiques. Mon consultant avait connaissance d'informations, notamment des rapports de l'époque, auxquelles je n'avais pas accès. Nous avons travaillé ensemble, puis j'ai continué les dialogues seule, mais avec les expressions qu'il proposait.

Le sous-titrage doit répondre à des contraintes linguistiques (texte fluide, clair, dont le registre correspond à celui du dialogue original) et esthétiques (il ne doit pas être trop prégnant, s'écrire sur deux lignes maximum, et proposer des césures logiques). Le sens doit être immédiatement compréhensible: le spectateur a souvent juste le temps de lire un sous-titre avant que s'affiche le suivant. Quand la formulation originale est complexe, la proposition traduite doit être plus simple. Par ailleurs, rendre un jeu de mots peut s'avérer difficile, a fortiori s'il est basé sur les sonorités; il faut alors trouver une solution qui provoque un effet comique même à la lecture muette, qui reste dans l'esprit et conserve la familiarité, voire la vulgarité de départ. Les accents sont également problématiques: on ne peut les transcrire, et on se tourne donc vers l'argot, des expressions particulières ou des élisions. Ces dernières sont cependant malaisées à lire, et on n'y recourt que lorsque l'accent est vraiment très appuyé dans la version originale.

On doit aussi retrouver le rythme et les appuis de l'original dans ce qu'on lit; dans la mesure du possible, on respecte donc l'ordre des mots que l'on entend - sans, bien sûr, dénaturer la sémantique du français. Le sous-titrage des chansons est un cas particulier. Le rythme, le nombre de pieds sont importants pour les accompagner le mieux possible, mais il faut toujours privilégier le sens: on ne va pas le sacrifier pour faire une jolie rime; ce serait gênant de lire un sens très différent de ce que l'on entend.

4. Damien Chazelle, 2018. 
En cours de travail, il marrive souvent de redécouper les sous-titres en fonction de ma traduction: si je trouve une formulation condensée pour traduire plusieurs idées, je peux choisir de rassembler deux sous-titres dits dans la foulée. Le découpage dépend aussi de la vitesse d'élocution: si l'acteur a un débit très rapide, il faut resserrer d'autant plus et respecter rigoureusement la lisibilité pour que le spectateur ne "coure pas après les sous-titres», alors que l'on peut traduire plus littéralement si le débit est lent.

Quand je suis satisfaite de mon sous-titrage, je l'envoie au laboratoire. Puis vient la simulation, la présentation du film sous-titré au directeur technique en ma présence. L'opérateur.rice de simulation aux manettes arrête le film dès qu'un problème survient: un texte trop long, une coquille, une incompréhension, un contresens. Je découvre à cette étape le film sur grand écran - et parfois la version en couleurs. Le sous-titre prend alors une importance supérieure; certaines choses sautent aux yeux, des détails qui nous avaient échappé, un décalage de rythme. On corrige les sous-titres en conséquence, on peut en supprimer ou les alléger parce que l'image suffit. La simulation dure entre deux heures et demie (un peu plus de la durée du film) et huit heures - et en moyenne trois à quatre heures. Elle peut être assez longue si le client n'est pas habitué et a la tentation de tout traduire; je dois alors expliquer que ce n'est pas nécessaire, et ces discussions prennent du temps. À l'issue de la simulation, les sous-titres sont "gravés dans la pellicule»-enfin, façon de parler: la version moderne de la copie d'antan s'appelle digital cinema package (DCP).

Au cinéma, une projection de contrôle est encore souvent prévue. Je vais alors chez Warner ou Paramount, par exemple, voir le film sur grand écran, seule ou dans le cadre d'une projection presse. Le plus souvent, les modifications ne sont plus possibles à ce stade: quand les journalistes voient le film, c'est que la sortie en salle est imminente. Chaque client a cependant son processus de travail, et avec Sony, je peux encore discuter et suggérer des changements. 


\section{Les étapes du doublage}

Le doublage répond à un processus différent. Tout d'abord, le film est pris en charge par un studio de doublage qui impose le logiciel avec lequel seront effectuées les différentes étapes. La première est la détection, qui consiste à décortiquer tous les dialogues du film et à noter sur une bande rythmo des signes qui correspondent aux mouvements de bouche. Tout d'abord, en début et en fin de phrase, une flèche vers le haut ou vers le bas indique qu'elle est ouverte ou fermée. Puis un trait horizontal pour les labiales M, B, P (il convient en effet de respecter celles qui sont très visibles à l'écran, ce serait gênant de ne pas en avoir en français à cet endroit-là), une croix pour les semi-labiales ( $F, V$, semi-fermeture), le symbole pour les ouvertures (é, i, a...), et un signe de liaison pour les fermetures (on, o, ou, u...). Enfin, un trait souligné plein indique que le personnage est hors-champ, et un souligné en pointillé qu'il est de dos ou qu'on ne voit pas sa bouche.

None of you cracker matherfuchers got no wark to da?
Bah alors, les visages pales, wous avegnien a glander?
Bah

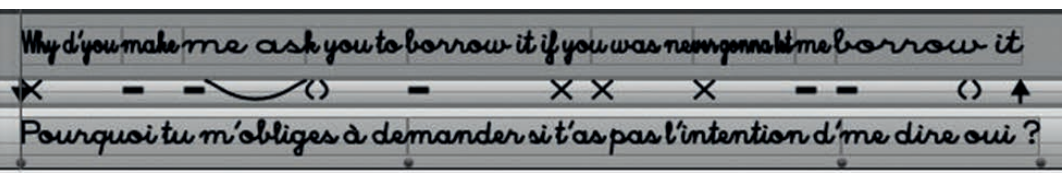

H's still shacked up with same chich smells of shit --

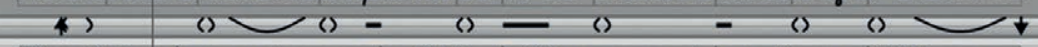

Il est toujours avec unep 'tite petasse qui pue le crot tin.

That Henry gecy threw that Albert guy out of his window this morning. Letype, la, Henry, il a jete, Albert par la fenstre, ce mation.

Exemples de bandes rythmo.

La détection progresse à raison de dix minutes de film par jour en moyenne. À l'issue du processus, le film est découpé en boucles d'une minute environ. Le traducteur les reçoit 
dans l'ordre, sous la forme d'un fichier informatique composé de l'image et de la bande rythmo.

Comme en sous-titrage, je regarde le film en entier avant de me mettre au travail. Quand je commence l'adaptation d'une réplique, j'écoute la version originale, je m'imprègne du sens et je cherche l'expression française qui va le rendre en respectant le plus fidèlement possible le rythme de départ et les labiales les plus visibles. Une phrase toute simple peut être très complexe à traduire quand son équivalent français n'a rythmiquement rien à voir. Souvent, la première idée qui me vient ne correspond pas à la «musique» de la phrase originale, elle n'est pas du tout synchrone. Je dois trouver une autre formulation en m'aidant de ce que je vois et de ce que je ressens, en veillant à ne pas m'éloigner de l'esprit et du sens de l'original. Les informations et les émotions doivent autant que possible se retrouver aux mêmes endroits. Je dois aussi tenir compte du jeu de l'acteur, d'une grimace ou d'un appui particulier sur un mot, qui sont autant d'éléments importants pour le comédien qui enregistrera la voix doublée. Ce dernier va "courir après la synchro», pour reprendre l'expression consacrée, si ces éléments ne sont pas respectés. Je vais souvent sur le plateau assister à l'enregistrement de doublages, même quand il ne s'agit pas de mes adaptations. Je trouve que c'est très formateur: cela permet de constater qu'un texte a beau être synchrone, le jover peut s'avérer très compliqué s'il a été trop trituré. Au début, je pensais trop au synchronisme et cela me bloquait. Avec le temps, j'ai acquis des automatismes et des habitudes, et aujourd'hui, certaines phrases me viennent beaucoup plus vite. Le plus simple est d'écrire ce qui vient instantanément, puis de le perfectionner; les labiales se mettent en place petit à petit. Mieux le doublage est fait, mieux on plonge dans l'atmosphère du film. Mais celle-ci varie toujours imperceptiblement: les comédiens français apportent immanquablement leur personnalité, même s'ils essaient de respecter l'émotion originale.

La réalisation d'un doublage est beaucoup plus longue que celle d'un sous-titrage: outre les dialogues essentiels à la compréhension, il faut en effet habiller le film et écrire des 
ambiances. Dans Seul sur Mars ${ }^{5}$, j'ai dû inventer des dialogues pour rendre le brouhaha dans la salle de contrôle de la NASA, en entremêlant des éléments techniques et des anecdotes de la vie quotidienne. Parfois, on s'échine à écrire une ambiance très documentée, que l'on n'entend plus une fois le mixage son effectué - mais il faut quand même la peaufiner. Cela peut être le bruit de fond d'une télévision qui diffuse un match de football américain au cours d'un repas où quinze personnes parlent; ou encore la météo, un jeu télévisé, les actualités, une foule, une manifestation. Il faut de plus respecter la synchro quand on distingue les bouches. Je constitue donc des collections d'ambiances que je peux éventuellement réutiliser d'un film à l'autre, surtout pour les sujets techniques; une scène de rue se fait plutôt à la volée. Les actualités sont un cas particulier, car elles doivent correspondre à l'époque du film. Je regarde beaucoup de séries françaises, par exemple des séries policières comme Le Bureau des légendes ${ }^{6}$, Braquo ${ }^{7}$ et Engrenages ${ }^{8}$, ou encore

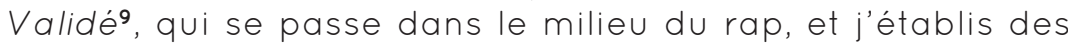
listes de vocabulaire, de jargon, d'expressions pour colorer mes adaptations. Quand j'écoute la radio, quand je regarde la télévision, j'attrape au vol une expression, un tic de langage des journalistes. C'est important d'être attentif à ces façons de parler, de grappiller dans la vie de tous les jours des expressions et de les consigner par thème, afin de donner de l'authenticité aux traductions.

Autre difficulté du doublage: la vitesse d'élocution. Quand un acteur parle très vite dans la version originale, il est préférable de demander au directeur de plateau si le comédien qui le doublera peut parler aussi rapidement - je saurai ainsi si je peux proposer un texte fourni, ou si je dois plutôt alléger. Il faut aussi se méfier de certaines expressions récurrentes ou d'automatismes de langage inexistants en français: pour les débuts de phrase hésitants en anglais, de type You know, Well, I mean, il convient de trouver une solution qui

5. Ridley Scott, 2015.

6. Éric Rochant, diffusée sur Canalt de 2015 à 2020.

7. Olivier Marchal, diffusée sur Canalt de 2009 à 2016.

8. Alexandra Clert, diffusée sur Canal+ de 2005 à 2019.

9. Franck Gastambide, diffusée sur Canalt en 2020. 
paraisse naturelle et ne sente pas la traduction. Cela peut parfois se régler en plateau, les comédiens trouvant spontanément des expressions auxquelles on n'avait pas pensé.

Il est bon de disposer d'au moins trois semaines pour le premier jet d'une adaptation. C'est un travail de longue haleine, plus physique que le sous-titrage. Comme le doublage est amené à être joué, il faut se mettre à la place du comédien qui parle à voix haute, lui donner un texte qui va l'aider à restituer les émotions. Je ne peux pas faire ce travail silencieusement; je parle, je m'exclame.

L'adaptation terminée, on passe à la vérification, qui s'effectue en présence du client et du directeur artistique. Je leur lis tous les dialogues du film en respectant le synchronisme, et eux regardent l'image ou vérifient que l'adaptation est fidèle à l'anglais. C'est une étape cruciale où chacun se rend compte si le texte est «jouable». À ce stade toutefois, on n'entend plus l'anglais; la veille, je me prépare donc chez moi en laissant la version originale en fond, pour me souvenir des émotions de telle ou telle scène, afin d'être la plus juste possible au moment de la vérification. C'est assez technique, car il faut être synchrone et dire le texte au moment où il passe derrière la barre rouge de la bande rythmo. La vérification est très importante, car elle permet de se rendre compte de ce que vit le comédien et de l'aider au mieux. Elle est souvent plus longue qu'une simulation de sous-titrage. Si d'emblée le doublage plaît à tout le monde, cela peut aller vite (trois heures). Quand le réalisateur est là, ça peut durer plus longtemps, car il a une idée précise des dialogues qu'il a envie d'entendre; en moyenne, il faut compter cina à six heures.

Une fois validé, le texte est enregistré par des comédiens. Ils sont convoqués un jour précis pour jouer les boucles dans lesquelles ils interviennent. Entendre son texte prendre vie, c'est le meilleur enseignement qui soit, et je conseille toujours aux adaptateurs qui commencent le doublage d'aller le plus possible en plateau. Ce peut être un peu dur pour l'ego d'entendre les comédiens ou un directeur artistique critiquer son texte, mais il faut s'y frotter pour se rendre compte de ce qui fonctionne ou non et ainsi progresser. Après l'enregistrement sur le plateau, le monteur replace les répliques au plus 
juste - c'est indispensable que la fin d'une réplique soit bien synchrone, car c'est ce que l'œil retient.

Vient ensuite l'interlock, une première projection de contrôle. Toutes les phrases ont le même niveau sonore, même les fameuses ambiances. L'ingénieur du son peut faire des merveilles, gommer des petites bulles dans la bouche, changer un mot en allant le chercher s'il a déjà été prononcé ailleurs. Si l'on décide de changements importants, on peut appeler le comédien pour faire un retake sur certaines phrases.

L'interlock est suivie du mixage son: les niveaux sonores, les filtres et les ambiances sont ajustés. Une nouvelle projection de contrôle a lieu, la double bande (autre vestige de l'époque où son et image étaient séparés). On finit de valider, et le film suit son chemin et sort en salle!

\section{Version sous-titrée vs version doublée}

La grande différence entre le sous-titrage et le doublage, c'est la transcription de l'oralité - toutes ces petites choses que l'on dit inconsciemment et qui rendent un texte plus vivant. Un texte de doublage comporte davantage d'élisions, d'expressions de type «euh» ou «tu vois».

VO:Where do youthinkyou'regoing alldone uplike afish supper? ST: Tu vas où comme ça, déguisée en sapin de Noël?

$V F$ : Bah dis donc, tu vas où comme ça, maquillée comme un camion?

Dans cette version doublée, le choix de l'expression "comme un camion» répond à la contrainte du respect des labiales de up et supper. Par ailleurs, l'interjection "bah dis donc», très orale, doit y figurer. Le comédien peut éventuellement employer ses propres expressions, avec l'accord du directeur artistique bien sûr. Il faut vraiment essayer d'imaginer ce que l'on dirait pour que ça paraisse naturel. Certains doublages sont très écrits, très littéraires, inutilement alambiqués, ampoulés, alors qu'ils devraient correspondre à la situation filmée - ou encore à l'époque où elle se déroule 
pour ce qui est des tics de langage. Pour le dernier Colette ${ }^{10}$, j'ai beaucoup lu avant de commencer mon adaptation, afin de m'imprégner de la langue de l'écrivaine et de celle de son époque; il faut se mettre dans la peau des personnages pour rejoindre au mieux le jeu des comédiens.

Parmi les expressions récurrentes, What the fuck are you talking about? peut se traduire de façon multiple selon les ambiances ou en fonction du fait que l'on voit le visage ou non - quand le personnage est de dos, on peut lui faire dire ce qu'on veut. En sous-titrage, je proposerais par exemple "C'est quoi, ces salades?», et en doublage, "Mais qu'estce que tu racontes, putain?!». On pourrait aussi adopter "Qu'est-ce que c'est que ce bobard?».

J'ai un lexique assez amusant pour rendre des expressions récurrentes, comme Jesus, oh boy, oh my, oh my God. Ca va de "Seigneur», "doux Jésus», «bonté divine», "pour l'amour de Dieu», "pour l'amour du Ciel» à «mince alors», «mais enfin», «saperlipopette», «sapristi», «sacrebleu», «juste Ciel», «fichtre», «nom d'un chien», «bon sang», "oh malheur», "oh punaise», "oh purée», "dis donc», "allons bon», «allons donc», «c'est pas vrai», «mais nan», «j'te jure», «sa race», «bordel de merde», «ras-le-bol», «la vache», «sans déconner». Je peux y piocher en fonction de la situation et du synchronisme. Évidemment, tout dépend du niveau de langue, on ne peut pas utiliser "saperlipopette» à toutes les sauces!

\section{Quelques aspects pratiques}

En cinéma, le paiement se fait à la bobine (soit environ dix minutes de film) en doublage, et au sous-titre en sous-titrage. Pour les projets destinés aux plateformes ou à la "vidéo», on est payé à la minute de programme, en doublage comme en sous-titrage, quel que soit le contenu: un film très bavard sera payé autant qu'un autre qui l'est moins. Au cinéma, on peut gagner entre 4000 et 6000 euros pour le soustitrage d'un long métrage; pour la durée équivalente d'une série, seulement 2500 euros. En 1993, à mes débuts, un seul

10. Wash Westmoreland, 2018. 
DESS préparait au métier d'adaptateur audiovisuel, à Lille. Aujourd'hui, on compte cinq ou six masters et, chaque année, une vingtaine d'étudiants arrivent sur le marché et acceptent souvent des projets très mal payés. On essaie de les mettre en garde pour que les tarifs ne dégringolent pas.

En ce qui me concerne, l'arrivée des plateformes n'a pas trop changé la donne pour l'instant. Je suis privilégiée, car nous ne sommes qu'une petite vingtaine de traducteurs à travailler uniquement pour le cinéma, sur 400 à 500 traducteursadaptateurs. Mais l'apparition du cinéma numérique a chamboulé les délais: il est désormais très facile de modifier le montage à la dernière minute. Au moment du Festival de Cannes par exemple, on travaille parfois encore la nuit précédant la projection d'un film, à la suite d'un changement de voix off. Certains ajustements peuvent aussi être induits par l'actualité. Dans un film qui sort prochainement, un personnage dit I can't breathe, la phrase qu'a prononcée George Floyd avant de succomber et qui a été reprise lors des manifestations Black Lives Matter en mai dernier. L'équipe de production cherche à présent une nouvelle réplique.

Les mesures de sécurité sont par ailleurs de plus en plus drastiques et peuvent devenir contraignantes. Pour certains gros films, je dois écrire les sous-titres en laboratoire, car la pellicule, extrêmement protégée, ne doit pas transiter par internet. J'ai même dû aller travailler directement au studio Deluxe à Los Angeles pour Le Bon Gros Géant de Spielberg. De son côté, Disney impose de travailler via un système en ligne auquel je me connecte avec des codes d'accès; cela me permet d'avoir l'image sans qu'elle soit physiquement sur mon ordinateur. Mais une fois que j'ai écrit les sous-titres, il ne m'en reste aucune trace...

Propos recueillis par Émilie Syssau.

mai.boiron@gmail.com www.maiboiron.com 
Après des études de cinéma à San Francisco, Maï Boiron fait ses premières armes chez Cinétitre à Saint-Cloud avant d'intégrer en 1994 l'équipe de Titra Film, puis de se lancer, en 2001 en tant qu'autrice dans la traduction et l'écriture de ses propres sous-titres, de I'anglais vers le français. Elle se met au doublage en 2014 .

\section{POUR ALLER PLUS LOIN}

Association des traducteurs et adaptateurs de l'audiovisuel (ATAA) : https://beta. ataa.fr/

Audiovisual Translators Europe (AVTE; fédération européenne des associations de traducteurs audiovisuels) : http://avteurope.eu/

Syndicat national des auteurs et des compositeurs (Snac), groupement doublage, sous-titrage, audiodescription: https://www.snac.fr/site/doublage-soustitrage/

Faire adapter une cuvre audiovisuelle - Guide du sous-titrage et du doublage, brochure éditée par l'ATAA: https://beta.ataa.fr/documents/Brochure_Ataa_version2019. pdf

CORNU Jean-François, Le Doublage et le sous-titrage. Histoire et esthétique, Presses universitaires de Rennes, 2014.

GALLO Serge, Traduction audiovisuelle, de l'ombre à la lumière, IDONEO Films, 2017. 